\title{
Heat Shields for Jet Engines
}

\section{PROTECTION BY LOW EMISSIVITY GOLD COATINGS}

\section{Kenneth Long}

Engelhard Industries, Sutton, Surrey, England
The very high reflectivity of gold-extending well into the infra-red range-is well known and is surpassed only by that of freshly polished silver. But as silver is readily tarnished, while gold remains unaffected by any atmospheric or corrosive attack, it is true to say that in practice gold, even in the form of a thin film or coating, is the most efficient reflector for infra-red radiation.

Many applications of this property are well established, but the advantages of gold as a very low emitter of radiation have not always been so fully appreciated.

The emissivity of a metal can be derived from the value of its reflectivity by the relationship

$$
\varepsilon=1-r
$$

where $\varepsilon$ is the emissivity and

$r$ the reflectivity on the scale $1.0=100$ per cent.
Thus a metal that is highly reflective will be a poor emitter, and a gold surface having a reflectivity of well over 95 per cent in the infra-red region will have a total emissivity of only 0.05 over a fairly wide range of temperatures. This compares with very much higher values for most constructional metals and alloys; for example stainless steel, if subjected to high temperature and therefore having an oxidised surface, gives a total emissivity of the order of 0.80 and will therefore radiate a very great deal more heat.

This inherent property of gold can be utilised by the application to metal surfaces of a coating of a preparation generally known as "liquid bright gold", Many variations are possible on the basic composition, but essentially these preparations consist of a complex sulphur compound of gold in an organic solvent, with small additions of readily oxidisable metals such

Part of a heat shield for one of the two Rolls-Royce Avon engines that powex the British Aircraft Corporation's "Lightning" being sprayed with a liquid gold preparation in the works of Lucas Gas Turhine Equipment. A simple heating operation then decomposes the organic constituents and produces a strongly adherent, continuous and dur' able film of gold

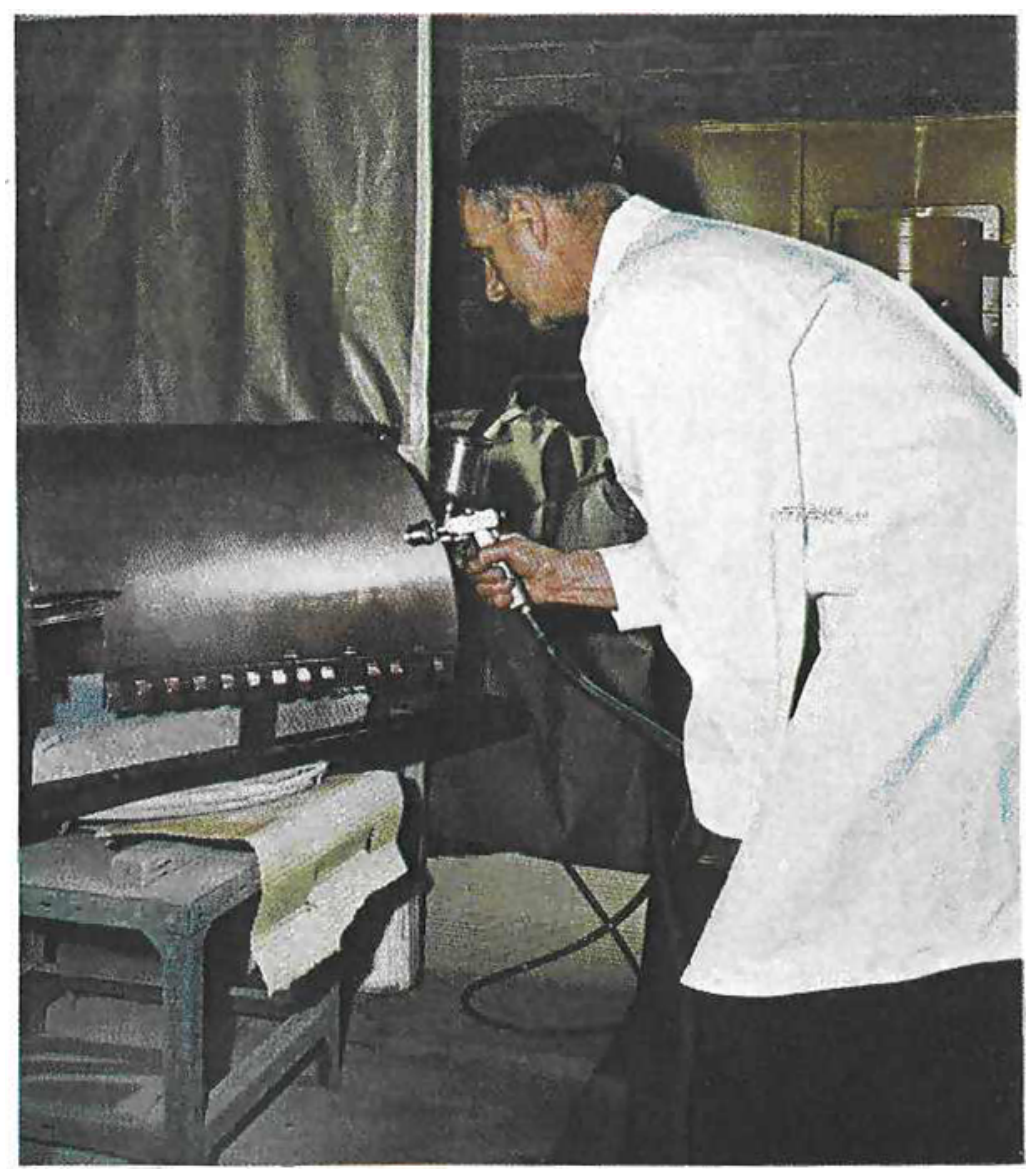




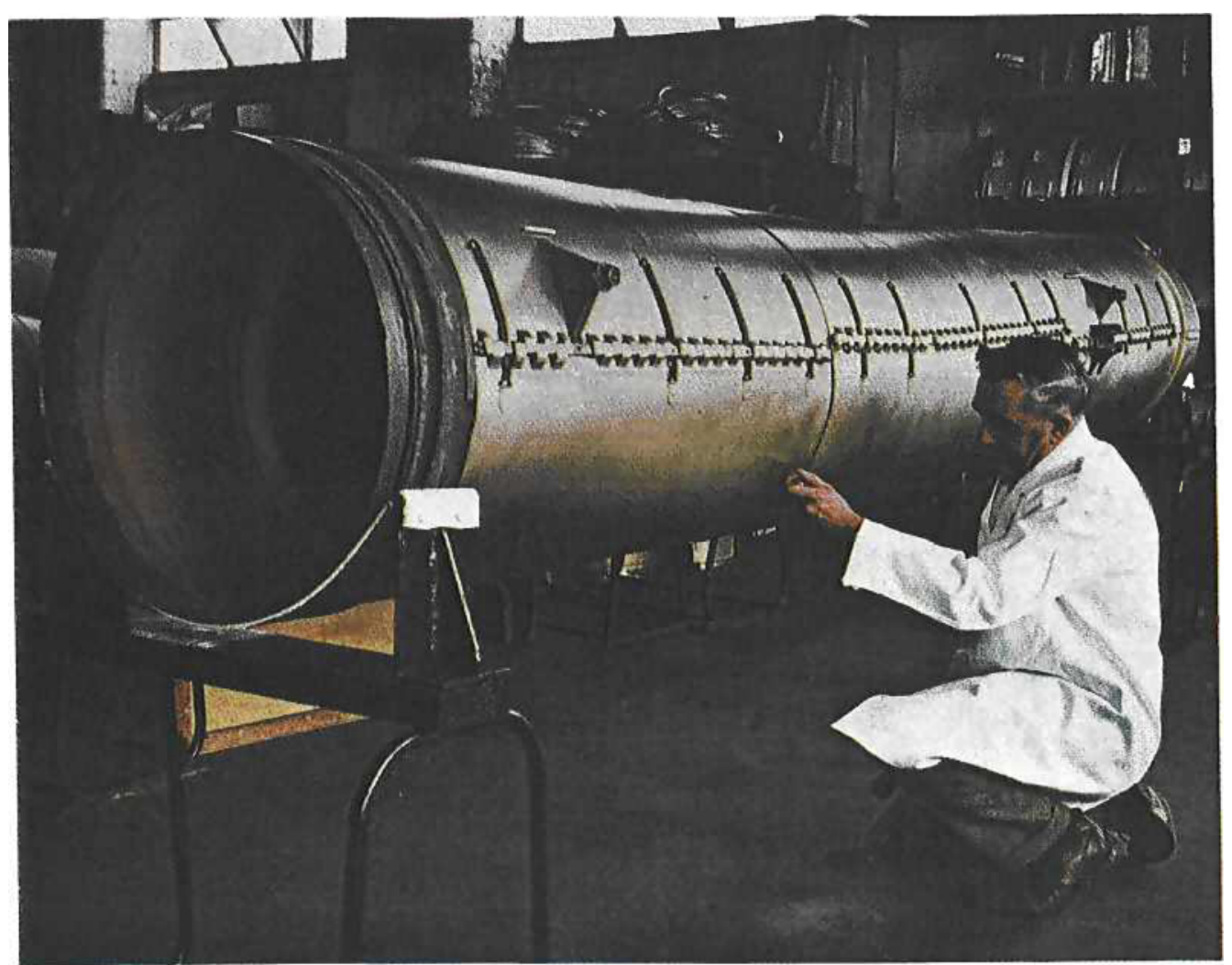

A completed heat shield for one of the jet pipes of the "Lightning". The low emissivity of the gold coating cuts down radiation from the hot jet stream and avoids overheating of the aircraft

as bismuth or vanadium to ensure good bonding to the substrate. These compositions can be produced with viscosities suitable for application by brushing, spraying or other means, and upon subsequent heating in an oxidising atmosphere they yield a firmly adherent film of gold.

For covering large areas, such as the heat shields on aircraft engines, spraying is preferred to ensure uniform coverage. The compressed air employed must be free from entrained water and dust, either of which could cause pinholes in the fired film, while the parts being coated must be clean and dry. After the coating has been applied it should be fired as soon as possible to avoid contamination. But given these few elementary precautions, a durable film of gold can readily be produced, some 5 millionths of an inch in thickness and of negligible weight. It will resist attack by jet fuel, hydraulic fluid or engine oil, and, provided that the substrate metal is itself unimpaired, will not suffer breakdown or exfoliation at temperatures up to $750^{\circ} \mathrm{C}$.

Among a number of applications to modern aircraft engines, perhaps one of the most interesting cases is that of the British Aircraft Corporation's "Lightning". This multi-role machine, designed for high altitude interception, reconnaisance or ground attack, and capable of speeds up to 1500 miles per hour, is powered by two Rolls-Royce Avon turbo-jet engines, joined by long jet pipes to fully variable reheat units. These are mounted one above the other on the centre-line of the aircraft to reduce the frontal area of the power plant, and the jet pipes run almost the whole length of the fuselage.

This versatile aircraft was originally designed for operation in the temperate climate of Great Britain, but it has for some years now also been in service in the heat and humidity of the Middle East and the Far East.

To avoid overheating problems it was decided at an early stage of the development of the aircraft to provide suitable heat shields, and this task was undertaken by Lucas Gas Turbine Equipment of Clitheroe, Lancashire, in co-operation with Rolls-Royce. It was quickly established that the gold film technique would solve the problem and immediate steps were taken to implement this decision.

Stainless steel shrouds were designed for the jet pipes, and were given a coating of an Engelhard liquid bright gold by spraying. The units were then given a firing cycle at $400^{\circ} \mathrm{C}$, and emerged from the oven with a closely adherent film of gold. A heatresisting blanket for the exhaust unit of the engine- 
This heat-resisting stainless steel blanket fitted to the exhaust unit of the Avon engine is also provided with a gold coating to reduce heat transfer

consisting of two layers of dimpled stainless steel with a filling of silicone fibre glass-was also treated in the same way and with equal success.

This application of the low emissivity property of gold has thus been completely successful in resolving overheating problems without the need for the extensive use of insulating materials.

In addition to this application in the "Lightning" jet aircraft, these gold coatings are being employed to reduce heat transfer on dragchute containers, tail cone assemblies and missile blast shields.

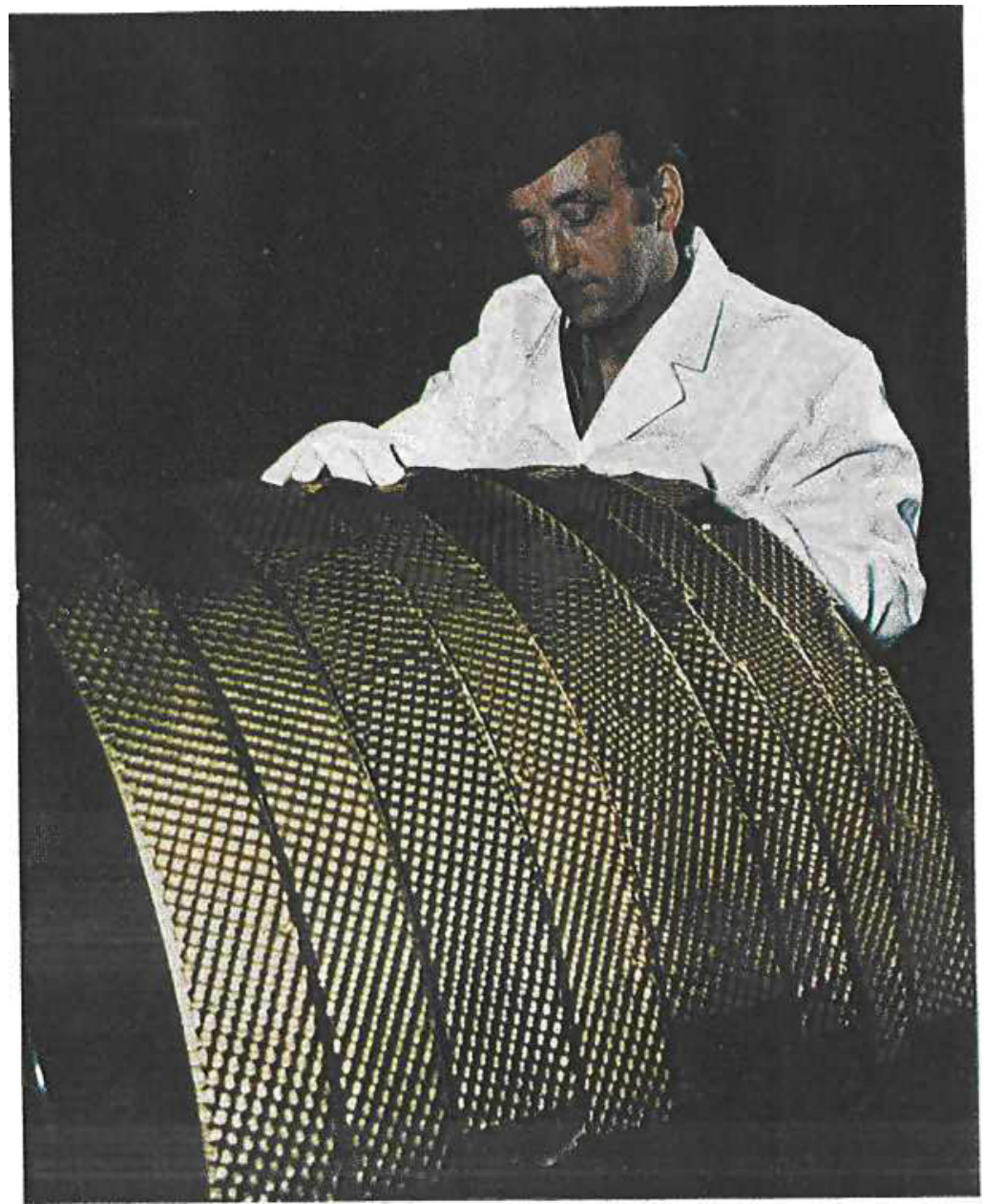

\section{Methods of Reducing Porosity in Thin Gold Electrodeposits}

The British Non-Ferrous Metals Research Association has recently been studying methods by which the residual porosity in thin gold electrodeposits may be reduced. Gold plating for electronic applications has to be reliably pore-free to ensure that even slight corrosion of the substrate cannot occur, particularly in the areas near contacts. A sufficient thickness of gold is therefore applied to ensure a very low density of pores.

The investigation has concentrated upon techniques which could be applied without undue difficulty to current plating procedures, and areas have been identified where an alteration in processing can result in a reduction in residual porosity. Two variables have been shown to be important in the consistent production of low porosity gold coatings. These are the original condition of the substrate to be plated and the method of agitation used during the electrodeposition process.

Surface features present on the substrate have been shown to affect significantly the porosity level of the electrodeposited gold coating. Inclusions have been identified as a source of residual porosity, but these can be removed by suitable cleaning processes. However, the surface layer of the substrate can be distorted due to working and, also, often contains depressions and craters which form a potential pore site. It has been found that the surface can be considerably improved by electropolishing prior to plating, and a procedure for producing a satisfactory substrate by electropolishing has been developed.

The effects of different methods of agitating the electrolyte during electrodeposition have also been studied. It has been found on a statistical basis that porosity can be reduced still further by utilising ultrasonic agitation during electrodeposition. A combination of the two processes, i.e. electropolishing and ultrasonic agitation, results in a consistently low residual porosity when thin $(2 \mu \mathrm{m})$ gold coatings are deposited.

It is hoped to study the causes for this slight residual porosity further and to apply these results on a larger scale in production plating shops.

I. R. A. C. 